\title{
A PPPR Road-map for the Plant Sciences: Cementing a Road-worthy Action Plan
}

\author{
Jaime A. Teixeira da Silva \\ P. O. Box 7, Miki-cho post office, Ikenobe 3011-2, Kagawa-ken, 761-0799, Japan \\ jaimetex@yahoo.com
}

\section{Doi:10.5901/jesr.2015.v5n2p15}

\begin{abstract}
This letter provides a step-by-step methodology for achieving post-publication peer review (PPPR) in the plant sciences. This involves identifying errors or other problems with scientific papers, linking the papers with their DOIs, and making queries and concerns publicly available at PubMed Commons and PubPeer. Using one concrete example, and screen-shots of the relevant sites, this methodology paves one possible viable road-map for achieving PPPR in the plant sciences.
\end{abstract}

Keywords: accountability; errors in the literature; post-publication peer review; responsibility

\section{Introduction}

This paper provides a set of practical step-by-step ideas of how to achieve effective, fair and responsible post-publication peer review (PPPR). PPPR allows for the retrospective analysis of the literature, in most cases to identify errors or problems that may exist in a scientific paper (Teixeira da Silva 2013a, 2014). Since the scientific integrity of a paper is what determines its use and validity, aspects that can on occasion be overlooked in the permeable traditional peer review system (Teixeira da Silva and Dobránszki 2015), PPPR then serves to cover those gaps. Ideally, the issues that are raised about a paper should be addressed by the authors, but in cases such as deceased authors, old papers in which emails are not available or where authors are no longer able to be contacted, that responsibility of addressing queries about papers is then passed onto the editors of a journal. This letter provides one set of parameters by which PPPR can take place in the plant science literature.

The first step of PPPR for its effective implementation must be the solid recognition that it is necessary, and important. The underlying objective must be solely to correct the literature using currently available tools to raise awareness. Unfortunately, there is a solid track record of the lack of responsiveness of authors and editors to requests to address issues in scientific papers, and most likely as a result of what appears to be a back-log in queries on scientific manuscripts, sites such as PubPeer and PubMed Commons have emerged. There is also a lack of culture of open discussion about the quality and problems of science publishers that extends beyond the limited and controlled interaction that occurs between authors and anonymous peer reviewers, or between authors and editors, during traditional peer review. If discussions about an already published paper have existed, these have usually taken place behind closed doors, within individual journal clubs. Apart from PubPeer and PubMed Commons, there are other commenting and open peer review systems such as f1000Research, ArXiv and Publons, but their dynamics of functionality and anonymity differ from PubPeer and PubMed Commons. The latter two were thus selected for the methodology explained next.

\section{Building the Road-map: Methodology}

Using PubPeer and PubMed Commons as the tools of choice for PPPR, next I present a simple case study as to how to most effectively achieve PPPR given currently available free tools.

1) Identify actual or possible errors in a scientific paper (e.g., Halmagyi et al. (2004); Fig. 1). This paper was selected based on the importance of cryopreservation in the biotechnology of chrysanthemum (Teixeira da Silva et al. 2015), one of the most important ornamental crops, and as part of a wider re-exploration of the literature of this plant through PPPR. Many papers now carry a digital object identifier (DOI) (Fig. 2). Preferably this should be done by a plant scientist in a plant science paper, and in more specialized fields, by specialists. This is not a sine qua non condition and most certainly the general plant scientist, or wider scientific public would be able to identify broad errors that may be worth correcting. 
2) Draft a list of the problems. Keep the comments as factual as possible. Avoid personal criticisms, or conjecture. Uncertainties, which are certainly an acceptable form of commentary, and form a core part of scientific Q\&A and dialogue, are also encouraged.

3) If the paper is listed on PubMed, then one has to first register with PubMed Commons. No anonymous comments are allowed. This allows for some quality control of the commentators, ensuring that an initial filter of trolls or possible sock-puppets is eliminated. It also filters through those scientists who are usually genuinely concerned, or who have genuinely valid claims that have not been addressed through traditional means, namely contacts to the authors and/or editor boards/publishers. In the case of PubMed Commons, the functionality is simple, and effective. There is comment moderation, thus eliminating the risks of libel, or slander. There is also a ranking system which shows how many readers found the comments to be useful (Fig. 3). Comments are posted immediately, and they are then relayed to PubPeer automatically (the case below was relayed in 3 days), using the identified scientist's name. In PubMed Commons, there is the possibility to invite each or all authors to the PubMed discussion, but one has to add the e-mails manually. There is no option to invite the journal editors to respond. Overall, it is a useful tool for anyone involved with research related with that topic because the comment appears very visibly below the PubMed Commons entry (Fig. 3). There is no option to link to PubPeer.

4) To make a PubPeer entry for a paper, the paper must have a DOI, but can also be identified with some other parameters such as title, or author's name. Most papers that appear on PubMed will have a DOI, but not all PubPeer papers that are linked via a DOI are linked on PubMed. Thus, PubPeer provides a more extensive commenting forum and platform than PubMed. If one enters a comment on a paper at PubPeer, one can register and usually registration takes place with a known link, such as an actual published paper, so there is veracity to the registration. However, a scientist can then choose to register anonymously, for example, as "Peer 20". Scientists also have the option of commenting anonymously, without requiring formal registration (Fig. 4). There is some heated debate at present about the risks and the advantages or disadvantages of anonymity within the framework of PPPR (e.g., Servick 2015). For now, and for the simplified purpose of this paper, until there is a clause that explicitly bans anonymity, I will consider anonymity to be a valid form of providing comments on a scientific paper at PubPeer. The comments, once posted at PubPeer, will usually be confirmed with a "Success" message and PubPeer claims that within approximately 24 hours, approved comments will be posted. There does appear to be some level of moderation at PubPeer, but this is also conducted by an unknown anonymous set of "peers" who are likely not trained in all disciplines. Thus, this is one weakness of PubPeer, but criticism of PubPeer lies beyond the scope of this letter because it is an issue still in debate. Needless to say that comments that are not posted have either been removed (i.e. not approved), or they have not posted properly (some technical error in posting of comments exists and commentators are advised to refresh their browser each time they enter a new comment). PubPeer comments are not linked automatically to PubMed, most likely because PubPeer allows for anonymous comments while PubMed Commons does not, i.e., the policies of both sites conflict with respect to the aspect of anonymity. PubPeer claims to contact the authors automatically with an alert. There appear to be errors and inconsistencies in the veracity of this claim. Journal editors are not contacted. After registering at PubPeer and at PubMed Commons, a set of five IDs exist: PMID (PubMed ID), DOI, PubPeer secure URL (https), PubMed Commons commentary with a URL, and a PubMed Commons-linked PubPeer secure URL (separate https), representing the culmination of points 1) to 4).

5) In many cases, such as a comparison of texts to show apparent duplicated text, to show how and where a figure may appear to be manipulated, where an apparent error in the text exists or to highlight any other issue, a visual representation usually gives the reader an excellent simple, and clear representation. An image can be created using a variety of softwares, and could be saved in a format such as .PDF, .jpg, or .png. A link to the figure file could be created on a personal web-page, blog, or any web-sites, always ensuring that user policies are respected. For this example, I selected imgur (http://imgur.com/) which allows for safe and registered or anonymous posting of images (Fig. 7) using simple functions like drag-and-drop. A web-site is created that can then be linked to the PubPeer or PubMed page.

6) It would be reasonable to assume that a month should be given for authors to provide a public response at either PubPeer or PubMed Commons, but preferably at both. If a scientist is busy, it takes a mere few seconds to at least drop a short note to indicate that they will respond as soon as possible to address the concerns and remedy the problems, if there are any. The assumption here is that a scientist is always 
responsible for their own literature. Failure to respond may represent a direct failure of that public responsibility although scientists should never equate silence with misconduct, which is determined by the research institutes and the publishers based on a set of evidence. The topic of academic and publishing misconduct lies beyond the scope of this paper.

7) Should the authors not respond, either because they did not receive the automatic alerts, because they are deceased, because they do not wish to respond, or for any other reason, it is then incumbent upon the journal editors to provide a response. For one simple reason. It is the editor board of a journal that approved, through peer review, the publication of a scientific paper. Thus, an editor in chief (EIC) and his/her editor board will always carry the responsibility of approving all papers under their watch for publication, as well as the papers approved by other EICs and other editors under previous editorial boards. It is a cumbersome task, and inconvenience even, but an absolutely necessary responsibility (Teixeira da Silva 2013b). Excuses by EICs and editor boards of being overloaded with work, that they are unpaid volunteers, or any other excuse is in fact inexcusable. For one simple reason: in most cases, especially those journals that are drawing direct benefit from systems such as the impact factor, is the assumption that such a system is based on a blemishfree and error-free literature, having followed rigorous peer review and editorial scrutiny. Thus editors must always be held accountable for the literature that they are supposedly safe-guarding. The emotive fact that one can empathize with the arduous task of being an EIC or editor, often voluntarily, does not serve as an excuse to be accountable for the literature in that journal.

As an example of how the system in steps 1-7 works, some queries about a chrysanthemum cryopreservation paper (Halmagyi et al. 2004) were made at PubPeer and PubMed Commons. The five important identifiers, as well as the original URL, are summarized in Table 1.

\section{Conclusions and Future Directions}

Questions that remain to be answered: Will the authors respond? If the authors respond, will they provide concrete solutions to what appears to be missing information in the methodology? If the authors do not respond, should they be recontacted directly? If the authors do not respond, should the EIC of the journal be contacted? If yes, anonymously, or not? If ultimately, the authors and the editors/publisher are contacted, and no response or solution is provided within a reasonable period of time (1-3 months), what action should be taken, and can anything be done to correct the literature? In the positive eventuality that the authors and/or the editors/journal move forward to provide a response and seek to correct the literature, what form does the correction take (erratum, corrigendum, expression of concern, retraction)? What happens if the authors and the EIC, editor board or publisher fail to address queries or concerns about scientific papers? Here are multiple issues related to the latter issue, but these also fall outside the scope of this paper.

In both PubPeer and PubMed Commons, there is one serious weakness: editors of the journals of the questioned papers are not automatically contacted, nor are any official publishing representatives. It is a weakness because it relies on the honesty and reliance of the authors to respond. A second weakness of PubPeer is that if one enters the DOI of the paper (e.g., 10.1007/s00299-003-0703-9 for the paper listed in this study; Table 1), only the registered or unregistered PubPeer entry is listed, and not the PubMed Commons-derived PubPeer entry, fortifying the need to complete a double entry on both sites.

This short paper serves to fortify the increasing need for PPPR in plant science as a result of an imperfect and permeable traditional peer review. Then, using a specific case, it highlights, in a step-by-step process, what needs to be done to achieve effective PPPR in a respectful and responsible manner, leaving the scientific community with no less than six official identifying parameters of that manuscript that should raise awareness among peers in that field of study, and seek accountability for correcting the literature in the hands of the authors and/or editors and/or publisher.

\section{Conflict of Interest Statement}

The author declares that the research for this paper was conducted in the absence of any commercial, financial or other relationships that could be construed as a potential conflict of interest. Any screen-shot images of proprietary material used under the fair-use agreement (http://en.wikipedia.org/wiki/Fair_use). 


\section{References}

Halmagyi A, Fischer-Klüver G, Mix-Wagner G, Schumacher HM (2004) Cryopreservation of Chrysanthemum morifolium (Dendranthema grandiflora Ramat.) using different approaches. Plant Cell Reports 22, 371-375.

Servick K (2015) Michigan judge asks PubPeer to turn over anonymous user information. Science DOI: 10.1126/science.aab0354

Teixeira da Silva JA. 2013a. The need for post-publication peer review in plant science publishing. Frontiers in Plant Science 4: Article 485.

Teixeira da Silva JA. 2013b. Responsibilities and rights of authors, peer reviewers, editors and publishers: a status quo inquiry and assessment. The Asian and Australasian Journal of Plant Science and Biotechnology 7(Special Issue 1): 6-15.

Teixeira da Silva JA. 2014. Postpublication peer review in plant science. Science Editor (Council of Science Editors) 37(2): 57+59.

Teixeira da Silva JA, Dobránszki J. 2015. Problems with traditional science publishing and finding a wider niche for post-publication peer review. Accountability in Research: Policies and Quality Assurance 22(1): 22-40.

Teixeira da Silva JA, Kim H-Y, Engelmann F. 2015. Chrysanthemum low-temperature storage, cryopreservation and synseed technology. Plant Cell, Tissue and Organ Culture 120(2): 423-440.

Table 1. Identifiers of papers that are subjected to post-publication peer review (an example for the plant sciences)

\begin{tabular}{cll} 
Identifier & Figure & Number or URL \\
\hline Original URL & Fig. 1 & http://link.springer.com/article/10.1007\%2Fs00299-003-0703-9 \\
DOI & Fig. 2 & $10.1007 /$ s00299-003-0703-9 \\
PMID & Fig. 3 & 13680136 \\
PMC & Fig. 3 & http://www.ncbi.nlm.nih.gov/pubmed/13680136 \\
PubPeer (anonymous) & Fig. 4 & https://pubpeer.com/publications/8D193B579573AC77319247AACA488F \\
PubPeer (PMC-linked) & Fig. 5 & https://pubpeer.com/publications/B8B319FBD9355D173EAEA4ABF49C37\#fb25748 \\
Altmetric & Fig. 6 & http://www.altmetric.com/details.php?citation_id=3723489 \\
Imgur & Fig. 7 & http:///imgur.com/TsgAE2r
\end{tabular}

DOI, digital object identifier; PMC, PubMed Commons; PMID, PubMed ID

Fig. 1 Screen-shot of original URL of Halmagyi et al. (2004).

Fig. 2 Screen-shot of DOl that may be found lower down the page on the original URL of Halmagyi et al. (2004).

Fig. 3 Screen-shot of Halmagyi et al. (2004) listed at PubMed with PubMed Commons comment posted immediately below it. Numbers in red: 1) Basic details of paper, including title, authors' names, journal name, volume and pages, as well as abstract. 2) PMID. 3) Link to the original site (Fig. 1). 4) The actual PubMed Commons comment, under a registered name. 5) The exact date and time the PubMed Commons comment was posted (same date in which authors are invited to respond). 6) A rating system of usefulness of comments (unclear who actually rates and how the rating takes place).

Fig. 4 Screen-shot of anonymous PubPeer comment about Halmagyi et al. (2004), which is identical to the PubMed Commons comment (but can be different, depending on the user's desired purpose). Numbers in red: 1) Basic details of paper, including title, authors' names, and journal name. 2) Link to the original site (Fig. 1). 3) The possibility to invite others to respond, but this requires registration (anonymous requests not allowed). 4) The actual PubPeer comment (anonymous, in this case, or as a registered user, named, or anonymous), including an equivaent of a thumbs up vs thumbs down rating, but this requires registration (anonymous requests not allowed). 5) Options to respond, add a new comment or make a report.

Fig. 5 Screen-shot of PubPeer comment with a separate URL that becomes posted automatically from PubMed, with the same features and functionality as anonymous or registered PubPeer comments (Fig. 4). Note (numbers in red): 1) the date of the transfer from PubMed to PubPeer (3 days in this case); 2) the date of the original posted comment at PubMed as a PubMed Commons comment. The date is important for accountability from authors and/or the EIC/editors/publisher.

Fig. 6 Screen-shot of Atlmetric page that one is directed to when pressing the "Social Shares" button of the Article Metrics, shown in Fig. 1. Clicking on the link shown there leads to the PubMed page (Fig. 2). The date of the comment is that of the original PubMed comment.

Fig. 7 Screen-shot of Imgur web-page showing the unique location for the .jpg file of the image showing a specific issue about a highlighted paper. The URL and unique address for the image is displayed on the web-browser (number 1). The image can also be conveniently linked to a range of popular social media sites (number 2). Scientists employing imgur (or any other site) to display images from published papers are recommended to always indicate the reference. The imgur (or other web-site address where the image can be found) should then be linked to the PubPeer sites (Fig. 4, 5), either together with the comment, or separately. 
Fig. 1.



Link to PubMed Commons comment

Fig. 2.

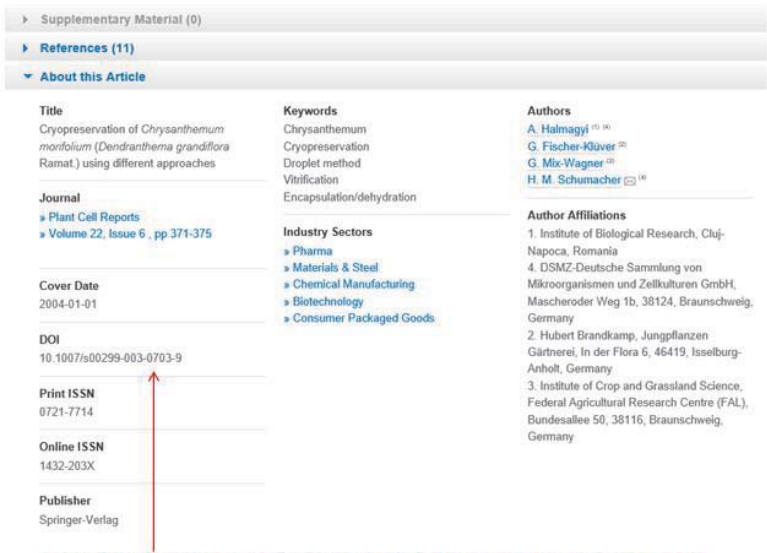

DOI which allows to link to PubMed Commons and PubPeer

Fig. 3

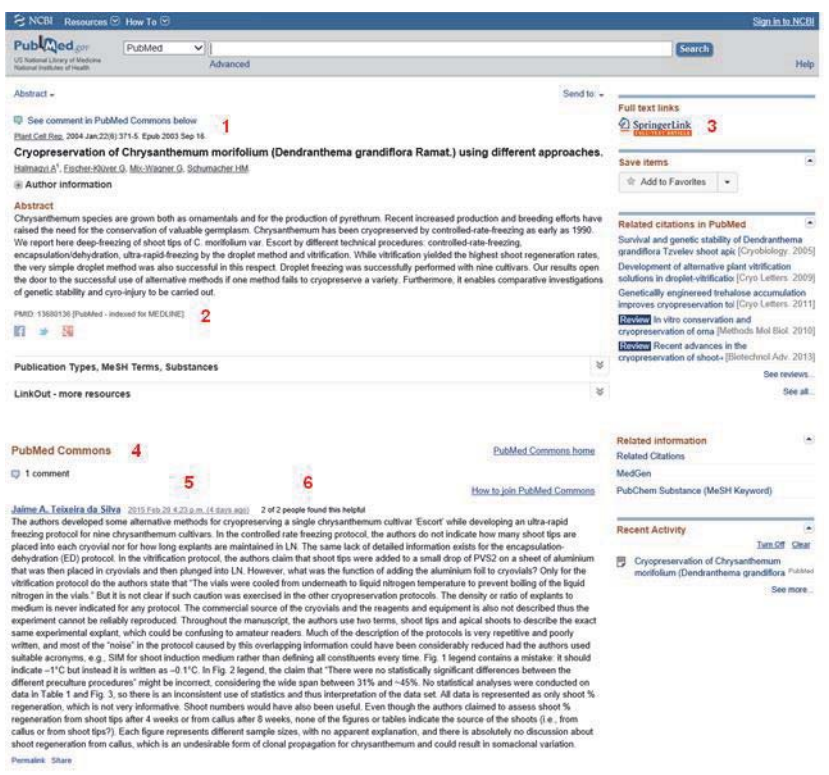


Fig. 4



Fig. 5

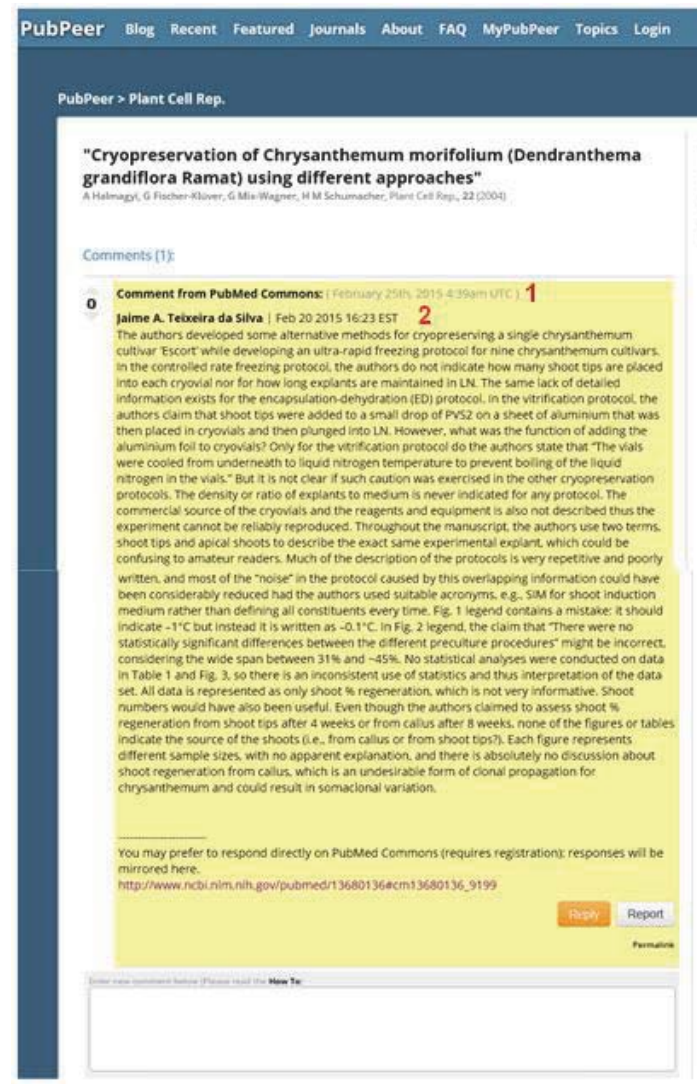

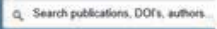

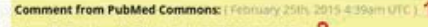
Jime A Teicedra da silva I reb 20.0151623 Est .

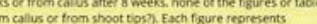

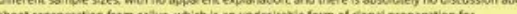
chosanthenum and coud resut in somacional variasion

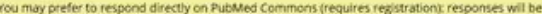

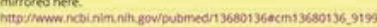
Dinvite obvers to the conversabon 
Fig. 6

O Altmetric

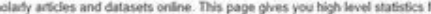

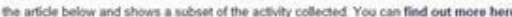

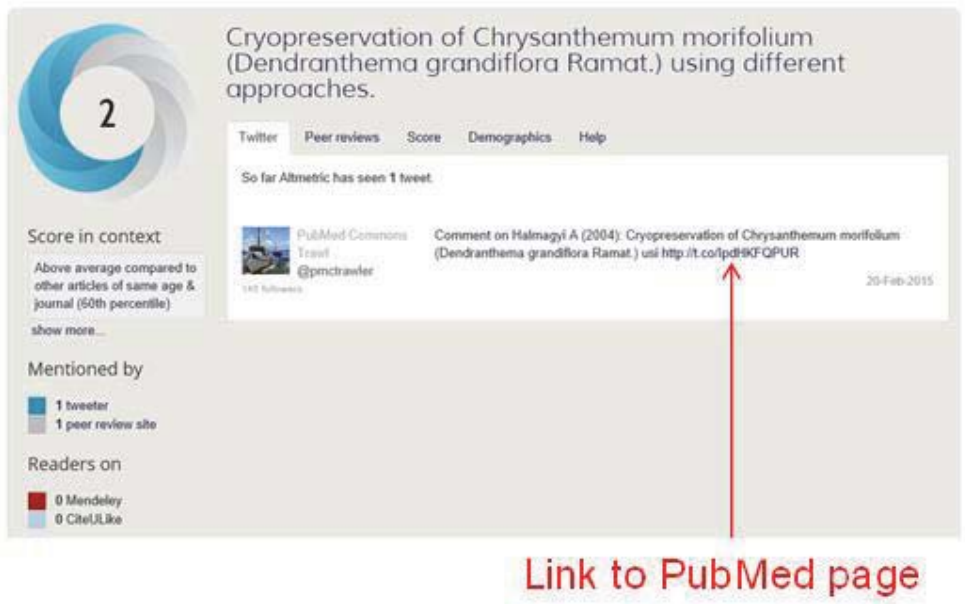

Fig. 7

\begin{tabular}{ll}
\hline http://imgur.com/TsgAE2r & $0-\mathrm{C}$ \\
\hline
\end{tabular}

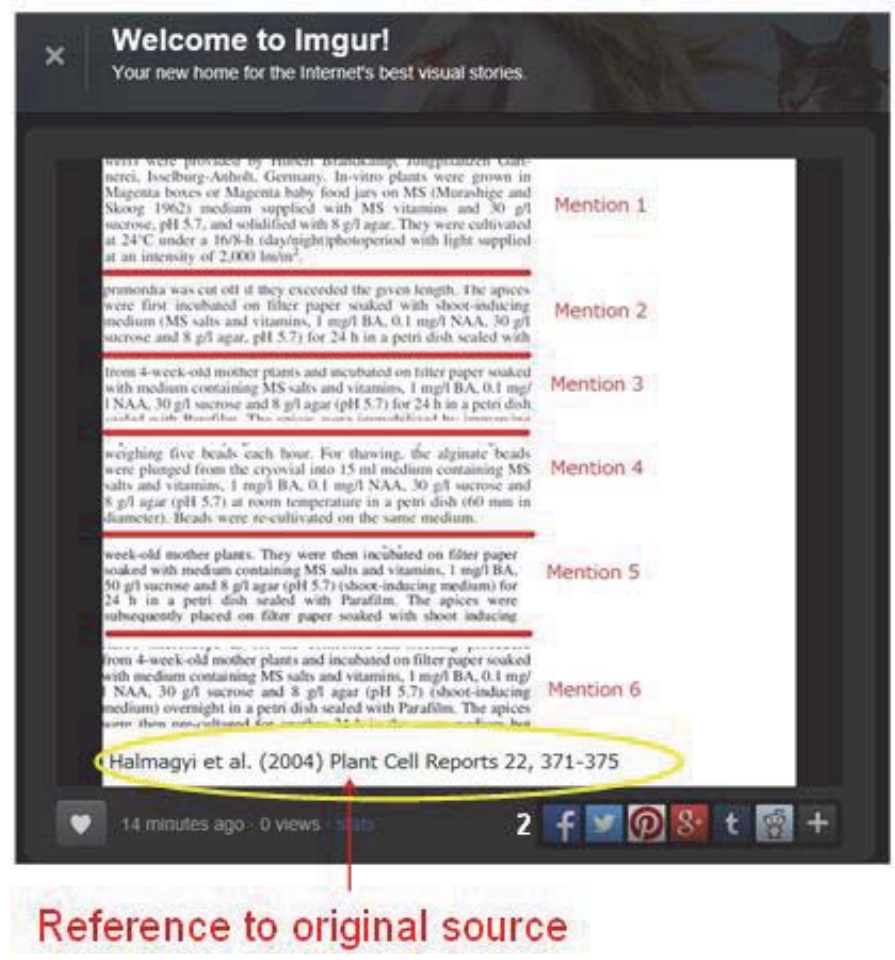


